Recepción: 09 / 07 / 2018

Aceptación: 17 / 09 / 2018

Publicación: 05 / 10 / 2018

Ciencias de la computación

Artículo de investigación

\title{
Análisis del uso de las Tecnologías de la Información y la Comunicación (TIC) como herramienta de la investigación científica
}

\section{Analysis of the use of Information and Communication}

Technologies (ICT) as a tool for scientific research

\section{Análise do uso de Tecnologias de Informação e Comunicação (TIC) como ferramenta de pesquisa científica}

\author{
Alexandra O. Pazmiño-Armijos ${ }^{\mathrm{I}}$ \\ apazmino_a@espoch.edu.ec \\ Jairo R. Jácome-Tinoco II \\ jjacome@espoch.edu.ec \\ Gloria E. Miño-Cascante ${ }^{\text {III }}$ \\ gmino@espoch.edu.ec \\ Mesías H. Freire-Quintanilla ${ }^{\text {IV }}$ \\ mefreire@espoch.edu.ec
}

Correspondencia: apazmino_a@espoch.edu.ec

\footnotetext{
${ }^{\text {I }}$ Magíster en Informática Empresarial, Especialista en Redes de Comunicación de Datos, Ingeniera en Electrónica y Computación, tecnólogo en Informática Aplicada, Docente de la Escuela Politécnica de Chimborazo, Riobamba, Ecuador.

II Magíster en Sistemas de Telecomunicaciones, Ingeniero en Electrónica y Computación, Tecnólogo en Informática Aplicada, Docente de la Escuela Politécnica de Chimborazo, Riobamba, Ecuador.

III Magister en Dirección de Empresas Mención Proyectos, Magister en Docencia Universitaria e Investigación Educativa, Doctor en Ciencias Económicas, Ingeniero de Empresas, Docente de la Escuela Politécnica de Chimborazo, Riobamba, Ecuador.

${ }^{\text {IV }}$ Magister en Seguridad Industrial Mención Prevención de Riesgos y Salud Ocupacional, Ingeniero de Sistemas Informáticos, Docente de la Escuela Politécnica de Chimborazo, Riobamba, Ecuador.
} 


\section{Resumen}

El uso de las Tecnologías de la Información y la Comunicación (TIC), constituye una herramienta para mejorar los procesos de investigación, por ello se llevó a cabo una revisión del marco conceptual y normativo de las TIC y su uso en la investigación, caracterizando el manejo vigente de las TIC durante el proceso de investigación. Se tipificó como una investigación descriptiva y de campo con un diseño no experimental. La muestra fue estratificada para 35 docentes investigadores que laboran en institutos universitarios. La recolección de datos se realizó mediante la aplicación de un instrumento tipo cuestionario con 5 escalas de medición y el tratamiento estadístico estuvo representado por la descripción de los elementos básicos y el análisis relativo frecuencial. Los resultados indicaron que los docentes utilizan las TIC al inicio de un proceso de investigación y algunas veces comunican por estos medios sus resultados. Entre sus conclusiones, se destaca el hecho en la cual los docentes tienen poco acceso a medios tecnológicos en sus sitios de trabajo.

Palabras clave: Tecnologías de la información y la comunicación; investigación científica; docentes; web.

\section{Abstract}

The use of Information and Communication Technologies (ICT) is a tool to improve research processes. Therefore, a review of the conceptual and normative framework of ICT and its use in research was carried out, characterizing the current management of ICT during the research process. It was typified as a descriptive and field research with a non-experimental design. The sample was stratified for 35 research teachers who work in university institutes. The data collection was done through the application of a questionnaire-type instrument with 5 measurement scales and the statistical treatment was represented by the description of the basic elements and the relative frequency analysis. The results indicated that teachers use ICT at the beginning of a research process and sometimes communicate their results by these means. Among its conclusions, it highlights the fact in which teachers have little access to technological means in their work places.

Keywords: Information and communication technologies; scientific investigation; teachers; Web. 


\section{Resumo}

O uso das Tecnologias de Informação e Comunicação (TIC) é uma ferramenta para melhorar os processos de pesquisa, por isso foi realizada uma revisão do quadro conceitual e normativo das TIC e seu uso na pesquisa, caracterizando gestão atual das TIC durante o processo de pesquisa. Foi tipificado como uma pesquisa descritiva e de campo com delineamento não experimental. A amostra foi estratificada por 35 professores de pesquisa que trabalham em institutos universitários. A coleta de dados foi realizada por meio da aplicação de um instrumento do tipo questionário com 5 escalas de medidas e o tratamento estatístico foi representado pela descrição dos elementos básicos e pela análise da frequência relativa. Os resultados indicaram que os professores usam as TIC no início de um processo de pesquisa e às vezes comunicam seus resultados por esses meios. Entre suas conclusões, destaca-se o fato de os professores terem pouco acesso a meios tecnológicos em seus locais de trabalho.

Palavras chave: Tecnologias de informação e comunicação; Investigação científica; professores; teia.

\section{Introducción}

Las Tecnologías de la Información y la Comunicación (TIC) cambiaron la forma en que nos relacionamos e interactuamos en todos los ámbitos de nuestra vida, los modos convencionales de interacción del tejido social modifican la forma en que adquirimos conocimiento, un ejemplo claro es el aporte de las TIC a la Investigación. Las TIC, explica Sancho (2006), han hecho mucho más efectivo el procesamiento y difusión de la información científica, permitiendo que investigar sea mucho más llamativo para docentes y profesionales; ya que amplía redes de conocimiento que son mucho más accesibles para la comunidad académica, a través de múltiples canales de información, sobre todo la internet.

El acceso a artículos científicos es mucho más abierto luego del auge de las TIC en la educación, y aunque aún muchos de éstos, se encuentran en redes privadas de universidades y centros científicos, en la web libre, indica Parra (2010), hay gran cantidad de documentos científicos de consulta abierta que permiten enriquecer la labor científica, y hacerla mucho más efectiva. Además, fomenta la retroalimentación de temas de investigación y la contrastación de resultados, contribuyendo en la formulación de proyectos de investigación que tendrán más incidencia en el 
campo de estudio, evitando repetir problemas ya estudiados, sino realizar un aporte a los mismos $\mathrm{u}$ otros por explorar.

Así mismo, ha permitido el fortalecimiento de redes de investigación, que se crean y mantienen en mayor contacto gracias a todas las opciones de comunicación que se encuentran disponibles y cuyo acceso resulta siendo fácil y económico. De esta manera, es que la labor del investigador se ve mucho más beneficiada con las herramientas que le brindan las TIC para realizar su tarea; instrumentos de indagación, software, páginas, programas e infinidad de herramientas han llegado para hacer mucho más fácil el acceso y procesamiento de la información que deriva en resultados contrastables y útiles en las etapas de investigación de las diferentes áreas académicas.

\section{Desarrollo}

La comunicación a lo largo de la Historia, ha sido fundamental en la vida del hombre, para poder relacionarse los unos y los otros. Para Según Belloch, C. (2012), dentro de las tecnologías de la información y la comunicación (TIC) se agrupan los elementos y las técnicas usadas en el tratamiento y la transmisión de la información, principalmente la informática, Internet y las telecomunicaciones.

Asimismo, Sánchez Vega (2011), indica:

En la actualidad, impera la capacidad y el poder de la televisión, Internet y la telefonía móvil, siendo los más poderosos en el mundo de la comunicación a distancia e instantánea. El uso de estas herramientas dejó de fundamentarse en la mera transmisión de información, para dejar paso a otra serie de innumerables causas, siendo las más importantes la diversión y entretenimiento, la enseñanza y el aprendizaje y la exploración social. [...] Obviamente, no podemos excluir los motivos académicos, puesto que la realización de los mismos a diferencia de años atrás, se llevan a cabo a través del ordenador, por lo que resulta imposible descartar este factor. (p.45)

Ante ello, es de destacar que la tecnología se ha desarrollado e integrado en la sociedad con mucha más celeridad en los países desarrollados que en los que se encuentran en vías de desarrollo, estableciendo en estos últimos, una fisura entre los cambios del antes y el después, en este sentido Serrano y Martínez (2003) expresan: 
Las nuevas tecnologías de la información han sido parte esencial de los recientes cambios dramáticos en la economía y la sociedad. Todos estos cambios han acentuado la separación (brecha) de los sectores sociales de bajos ingresos respecto de aquellos con mayores ingresos y con posibilidades y opciones de acceso a la información. Solo un bajo porcentaje de la población mundial ha sido beneficiado de las bondades de la tecnología y solo unos cuantos son los que tienen acceso a toda la gama de servicios que esta ofrece. Esta condición es conocida como la brecha digital. (p.13)

En este sentido, es de indicar que el mundo de la tecnología está presente en casi todos los hogares, constituyéndose el mundo de las nuevas tecnologías un componente importante en nuestra conexión con el mundo y con las personas que nos rodean y con las que queremos comunicarnos.

\section{Las TIC como herramienta de investigación}

En el entendido, que el apego de las TIC en investigación se ha considerado de vital uso, pues logra romper los paradigmas que la niegan dado su apoyo para facilitar el acceso a fuentes especializadas de información y conocimiento, en la materia de investigación. En este sentido Area (2002), explica algunas ventajas y desventajas, tales como:

\section{Ventajas}

Las TIC suponen una herramienta única, rápida y eficaz, la cual disminuye mucho los costes de investigación y permite una gran manejabilidad en cuestión de datos.

Permite a través de programas y aplicaciones, estudiar la información de una forma más dinámica y en menos tiempo.

Ofrece mayor comunicación entre profesores y alumnos al momento de consolidar los procesos de investigación, permitiendo el trabajo de forma cooperativa y con un alto grado de interdisciplinariedad.

Permite la visualización de simulaciones para aprender con menos tiempo de forma atractivo, con la disposición de acceso a múltiples recursos educativos y entornos de aprendizaje 
Favorece el desarrollo de habilidades de búsqueda y selección de información digital mejorando las competencias de expresión y creatividad de los investigadores universitarios.

Prevé la duplicidad de trabajos de investigación, ante la facilidad de la consulta inmediata de antecedentes de trabajos relacionados de forma similares y/o en campos similares.

Mediante el uso de las TIC, los investigadores acceso a Internet cualquier persona puede estar informada acerca de las últimas novedades de prácticamente cualquier tema. Esta es una gran ventaja ya que no limita el conocimiento a un libro de texto o un docente dentro del salón, además de que se pueden contrarrestar fuentes y opiniones y llegar a distintos puntos de vista sobre un asunto.

\section{Desventajas}

- Las TIC, como una fuente inagotable de conocimiento, lo es en igual medida de distracciones. Es muy fácil que con esta herramienta surjan pérdidas de tiempo constantes, por lo que cada investigador debe autocensurarse en estas cuestiones y dejar las distracciones de internet para los ratos de ocio, evitándolas al máximo cuando se está investigando.

- Existe la posibilidad de obtener información no confiable y de procedencia dudosa que coloca en vilo las teorías utilizadas durante un proceso de investigación.

- El uso excesivo de la TIC puede anular el pensamiento crítico, ya que muchos esperarán encontrar en la web todas las respuestas a los dilemas investigativos que se presentan dejando de un lado la reflexión personal.

\section{Metodología}

La presente investigación se tipifica como descriptiva de campo. De acuerdo a lo establecido por Méndez (2012), estos estudios se realizan en función de la identificación de elementos que intervienen en el problema; es decir, delimitando los hechos posibles que están vinculados en el uso de las TIC como herramienta de investigación. El proceso de investigación de campo, se desarrolló partiendo del conocimiento de los hechos, de la unidad de estudio y de los factores que intervienen en la realidad sin establecer control alguno. (Arias, 2014). El diseño fue no experimental, definido según Hernández, Fernández y Baptista (2014), como aquellos estudios 
que permiten establecer la perspectiva y comportamiento general de una variable en grupos de personas en un momento determinado, sin manipular deliberadamente la variable. Se utilizó un muestreo estratificado de 35 docentes investigadores que laboran en institutos universitarios. La recolección de datos se realizó mediante la aplicación de un instrumento tipo cuestionario con 5 escalas de medición (siempre, casi siempre, algunas veces, casi una y nunca). El tratamiento estadístico estuvo representado por la descripción de los elementos básicos y el análisis relativo frecuencial.

\section{Resultados}

Posterior a la aplicación del instrumento a los 35 docentes investigadores, se obtuvieron los siguientes resultados:

- En cuanto al uso de las TIC como herramienta de investigación científica, se pudo evidenciar que casi siempre los docentes las utilizan al inicio de un proceso de investigación, específicamente en las consultas de los foros virtuales, video beam, diapositivas, weblog y contenidos teóricos y de multimedia. Asimismo, se develó que casi nunca utilizan las video conferencias y el webquest y siempre consultan los contenidos para ampliar el conocimiento de lo investigado.

- A veces los docentes investigados utilizan las TIC para comunicar los resultados de sus investigaciones. Por otro lado, se constataron docentes que no manejan los medios tecnológicos por no tener absceso ni disponer de sistemas computarizados.

- En relación a las habilidades y destrezas para utilizar la TIC en los procesos de investigación, se infirió que los docentes están provistos solo de algunos conocimientos para lograr con eficiencia el uso de las TIC, a pesar de observarse que un gran número de ellos no manejan estos medios con una alta experiencia.

Por otro lado, se confirmó que solo a veces navegan en Internet como medio de investigación, por no tener a la disposición de un computador personal, sumado a la no existencia de este medio en las instituciones donde laboran y hacen investigación. Todo ello, a pesar de indicar una actitud de aceptación para trabajar bajo el sistema operativo Windows y hacia el uso regularmente del correo electrónico. 


\section{Conclusiones}

Existe poco acceso por parte de los docentes a medios tecnológicos en sus sitios de trabajo, dada que las instituciones cuentan con la tecnología adecuada y equipos actualizados, pero no posee la dotación suficiente para satisfacer los requerimientos académicos e investigativos de los docentes.

Por otro lado, se percibió la no disposición de laboratorios computacionales ni personal especializado para mantener cursos de formación tecnológica para el personal, específicamente aquellos que realizan actividades de investigación. Sin embargo, dentro de las políticas institucionales, está el conceder licencia para aquellos docentes interesados y de la cual muy poco la solicitan, a pesar que muchos docentes poseen una actitud favorable en cuanto a considerar que las TIC constituyen una valiosa herramienta para los procesos de investigación.

Un alto porcentaje, señalo la necesidad de actualizarse en cuanto al manejo del software libre, para facilitar la búsqueda de material teórico y herramientas técnicas que beneficien la producción investigativa en la institución a través del fomento y práctica de los valores de cooperación y solidaridad mediante el uso de las TIC. 


\section{Referencias bibliográficas}

Area, M. (2002) Sociedad de la Información, Tecnologías Digitales y Educación. Publicación online. Disponible en http://tecnologiaedu.us.es/bibliovir/pdf/tema1.pdf

Arias, F. (2014). El Proyecto de Investigación. Guía para su elaboración. 2da. Edición. Caracas: Episteme. C.A.

Belloch, C. (2012). Las Tecnologías de la Información y Comunicación en el aprendizaje. Material docente [on-line]. Departamento de Métodos de Investigación y Diagnóstico en Educación. Universidad de Valencia. Disponible en http://www.uv.es/bellochc/pedagogia/EVA1.pdf

Hernández, R. Fernández, C. y Baptista P. (2014) Metodología de la Investigación. México: McGraw - Hill Interamericana de México, S.A.

Méndez, C. (2012). Metodología Económica, Contable, Administrativa. Santa Fe de Bogotá, Colombia: McGraw - Hill Latinoamericana.

Area, M. (2002) Sociedad de la Información, Tecnologías Digitales y Educación. Publicación online. Disponible en http://tecnologiaedu.us.es/bibliovir/pdf/tema1.pdf

Parra Castrillón, E. (2010). Las redes sociales de Internet: también dentro de los hábitos de los estudiantes universitarios. Anagramas: Rumbos y sentidos de la comunicación.

Sánchez Vega, E. (2011). El uso de las TIC; un hábito actual de los estudiantes universitarios. Buenas prácticas con TIC para la investigación y la docencia (pp. 1-6). Málaga: Universidad de Málaga.

Sancho, J.Ma (Coord.)(2006) Tecnologías para transformar la educación. Ed. Alkal. Madrid.

Serrano, A., y Martínez, E. (2003). La brecha digital: mitos y realidades. México: Universidad Autónoma de Baja California. Recuperado de: http://books.google.es/books?hl=es\&lr=\&id=nw8PLfm4Ma4C\&oi=fnd\&pg

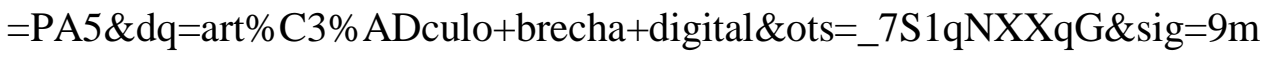
CL0UmdytqzjDllQtwSJaiMiX4\#v=onepage \&q=art\%C3\%ADculo\%20brec ha\%20digital\&f=false 
Análisis del uso de las Tecnologías de la Información y la Comunicación (TIC) como herramienta de la investigación científica

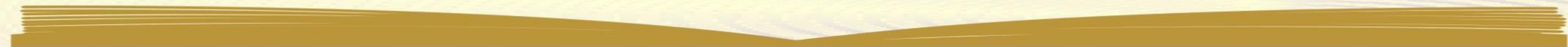


Análisis del uso de las Tecnologías de la Información y la Comunicación (TIC) como herramienta de la investigación científica

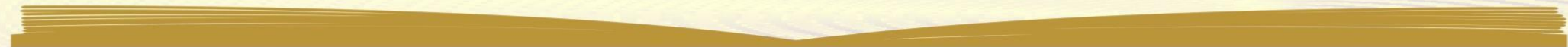


